\title{
A Stochastic Optimal Control Model with Internal Feedback and Velocity Tracking for Saccadic Eye Movements
}

\author{
V. Varsha ${ }^{\mathrm{a}, *}$, Aditya Murthy ${ }^{\mathrm{a}, \mathrm{b}}$, Radhakant Padhi ${ }^{\mathrm{a}, \mathrm{c}}$ \\ ${ }^{a}$ Centre for Biosystems Science and Engineering, Indian Institute of Science, Bangalore 560012, India \\ ${ }^{\mathrm{b}}$ Centre for Neuroscience, Indian Institute of Science, Bangalore 560012, India \\ ${ }^{c}$ Department of Aerospace Engineering, Indian Institute of Science, Bangalore 560012, India
}

\section{A R T I C L E I N F O}

\section{Keywords:}

Stochastic optimal control

Internal feedback

Velocity tracking

Saccadic eye movement

\begin{abstract}
A B S T R A C T
A new stochastic optimal control model with internal feedback and velocity tracking is presented in this paper to study saccadic eye movement control. Recent evidence from neurophysiological studies of superior colliculus suggests the presence of a dynamic input to the saccade generation system that encodes saccade velocity, rather than just the static information of the desired saccade amplitude and direction. The new evidence makes it imperative to test if the saccadic control system can use a desired velocity input to achieve accurate behavioral outcomes. To test such a velocity-based architecture of saccade control, a new optimal control tracking model that incorporated two unique characteristics of internal feedback and stochasticity has been developed. The proposed model was validated using behavioral data of saccades generated by healthy human subjects in an experimental setup. The model was able to generate displacement and velocity trajectories of horizontal saccades made to different amplitudes and predict saccades made to vertical and oblique directions. It captured the main sequence relationship between saccade amplitude and peak velocities that were observed in the behavioral data. This paper presents a novel optimal control framework with tracking and internal feedback and proposes the first-ever model of the saccadic system that uses an alternate interpretation of velocity-based control, contrary to the dominant end-point based models available in the literature.
\end{abstract}

\section{Introduction}

Saccades are very fast and accurate movements. Since they are rapid, saccades cannot make use of sensory feedback information about the state of the eye for its precise execution [1]. Therefore, they are thought to be largely pre-programmed to achieve the goal of landing the eye on the target. This idea of pre-programming the control has been realized using the framework of optimal control theory, which has been extensively used to understand the control of biological systems. The motivation of using optimal control framework in biological systems is that evolution has led these systems to converge on the best possible way to accomplish efficient performance. Hence, these models try to address the central problem of selecting the biologically relevant costs and constraints that can explain observed behaviors. Optimal control models have been successfully used to understand the basis of varieties of sensorimotor behaviors like locomotion, reaching, saccades, etc. (discussed in $[2,3])$.

The earlier optimal control models of the saccadic system, based on feed-forward control, suggested that the cost that is being optimized is the time taken for making the saccade and such a time-optimal controller gave rise to bang-bang control [4]. However, these models failed to capture many aspects of the observed saccade trajectories and the motor command. Another drawback of the early models was that they considered the saccadic system to be deterministic even though it is well established that noise is an inherent feature of the motor system [5]. The stochastic nature of the system reflects at the output as behavioral variability which has been established by many studies that quantify the variability in saccade metrics such as the saccade main sequence, peak velocity of saccades as well as saccade trajectories [6-10]. All the empirical evidence suggests that models that do not incorporate stochasticity are incomplete.

More recent optimal control models have however incorporated the presence of noise. Such a stochastic optimal control framework has two prominent philosophies of saccade control: feed-forward control and internal feedback control. Feed-forward control models generate the optimal control signal from static information regarding the goal of the

\footnotetext{
* Corresponding author.

E-mail addresses: varshav@iisc.ac.in (V. Varsha), adi@iisc.ac.in (A. Murthy), padhi@iisc.ac.in (R. Padhi).
} 
movement. In the case of saccades, an example of such a model is the speed-accuracy trade-off model that minimizes a combined cost of the duration of saccade along with the variance in the endpoint suggesting that the saccade kinematics are generated such that an optimal trade-off between its speed and accuracy is achieved [11]. Signal-dependent noise was incorporated in the model as an extra additive signal acting on the optimal control signal. This model was able to capture the main sequence saccade characteristics well but failed in the case of perturbed saccade trajectories.

Saccade perturbation studies suggested that a moment-to-moment control through sensory feedback is important. However, the saccadic system is devoid of such a feedback control due to the rapid time course that precludes any sensory feedback information from being relevant. One possible solution is to use non-sensory feedback and there exists much experimental evidence suggesting the same. The earliest evidence of a non-sensory feedback loop, also known as internal feedback, affecting eye movements was suggested through perturbation experiments in monkeys [12]. The study found that although saccades were interrupted by stimulation mid-flight and target information was made unavailable, the interrupted saccade foveated the target accurately on the removal of the interrupting stimulation. The existence of such internal feedback has also been confirmed by other studies using different saccade tasks that showed online correction of saccade trajectories [13]. Further, a transcranial magnetic stimulation (TMS) study showed that a TMS perturbed saccade trajectory was corrected within the same saccade to produce accurate movements to the target without relying on visual input [14]. This study claimed that the oculomotor commands are monitored as they unfold via an internal feedback mechanism and are corrected for perturbation.

The ability to monitor and control saccadic motor commands in realtime using internal feedback was incorporated using an extension of the optimal control approach called the optimal feedback control [15]. The theory proposes that the control signal is generated at each time step based on the estimate of the current state of the system, using a forward model present in the internal feedback loop. Since eye movements cannot make use of sensory feedback, it is hypothesized that the real-time estimate of the state is obtained through the forward model, using a copy of the control signal given to the muscles. One such model in the optimal feedback control framework proposed a controller that penalizes the control effort, the duration of movement, and the error of the states from the desired final state. The final states were regulated about the desired saccadic displacement to be achieved by the eye and zero final velocity. The model could explain both nominal saccade trajectories and saccadic behavior during adaptation $[16,17]$. The control signal was generated dynamically using a displacement error which was obtained using the desired input of final displacement and the prediction of instantaneous displacement obtained through the forward model that was part of the internal feedback. However, given that the forward model predicts both displacement and velocity information, an alternate possibility that arises is a dynamic control based on velocity information. But this would require that the saccade kinematics in the form of the desired velocity be dynamically encoded by superior colliculus (SC) neurons which are thought to provide the desired input to the saccadic controller. This hypothesis is supported by a recent neurophysiological study that showed a robust correlation of instantaneous activity of single neurons in the superior colliculus to the instantaneous eye velocity throughout the saccade duration during normal as well as blink-perturbed saccades [18].

In summary, the existing optimal control models of saccadic eye movement are all predominantly based on the philosophy of endpoint control that uses a static desired input of final saccadic displacement to produce the optimal control signal $[11,17,19]$. The possibility of a tracking control based on dynamic input information of velocity has not been explored despite the evidence that the superior colliculus which provides desired input to the saccade execution system encodes the saccadic velocity [18].
To address this caveat, in this study, an optimal controller that can use a dynamic velocity signal as desired input for saccade generation is developed. Two major features that were suggested by experimental studies of the saccadic system, namely the use of internal feedback [12, $14]$ and the presence of stochasticity $[20,6]$ has also been incorporated in the proposed model. This work presents the first-ever optimal control model schema with internal feedback that generates the control signal that allows tracking of the desired velocity in the presence of noise. It is also imperative to test the ability of such a new control mechanism to capture the performance of the actual saccadic system and hence the behavioral data of saccades were collected from healthy human participants. The model is shown to be capable of explaining the saccadic behavior with high accuracy. It captures the trajectories as well as the main sequence relationship exhibited by the saccadic system across multiple amplitudes. The model also predicted the trajectories of saccades made to different directions.

\section{Proposed Velocity Tracking Model with Internal Feedback}

A velocity tracking stochastic optimal control model with internal feedback is proposed for saccade generation. The goal of the model is to track a desired saccade velocity. The average behavior across repetitions is assumed to be the optimal human behavior and hence the desired velocity input for each individual is modeled as the mean velocity of all saccades produced by the individual to each target during the experiments. An optimal control signal is designed for tracking the desired velocity using an estimate of the state from a forward model (described in Section 2.3). The control signal is assumed to be corrupted by signaldependent additive noise and its characteristics are discussed in Section 2.2. The noisy control signal is the input to the oculomotor plant that generates the saccade trajectories (described in Section 2.1). A schematic block diagram of the model is given in Fig. 1 and the details about the different blocks constituting the model are described in the following subsections.

\subsection{Oculomotor plant dynamics}

The oculomotor plant is modeled as a lumped system consisting of the extraocular muscles and eyeball together and is represented by the block labeled as the plant in Fig. 1. A second-order spring-mass-damper model which provides a good approximation of this system has been used to derive the plant dynamics using a simple force balance equation [21]. The input to the dynamic system is the firing rate produced at the motor neurons innervating the oculomotor muscle and it is assumed to be equivalent to the torque required to move the eye. If the input firing rate is represented by $F(t)$, the force balance equation can be written as

$F(t)=J \ddot{\theta}(t)+B \dot{\theta}(t)+K \theta(t)$

where the moment of inertia, viscous coefficient, and elastic coefficient of the eyeball system is given by $J, B$, and $K$ respectively. This equation can be represented as a continuous linear dynamical system in statespace form as

$\dot{x}(t)=A_{c} x(t)+B_{c} u(t)$

where $u(t)$ is the control signal defined as equivalent to the firing rate $F(t)$. The state $x(t) \triangleq[\theta(t), \dot{\theta}(t)]^{T}$ and state matrices $A_{c}$ and $B_{c}$ are given by

$$
\begin{aligned}
& A_{c} \triangleq\left[\begin{array}{cc}
0 & 1 \\
\frac{1}{\tau_{1} \tau_{2}} & -\left(\frac{\tau_{1}+\tau_{2}}{\tau_{1} \tau_{2}}\right)
\end{array}\right] \\
& B_{c} \triangleq\left[\begin{array}{c}
0 \\
\frac{1}{\tau_{1} \tau_{2}}
\end{array}\right]
\end{aligned}
$$




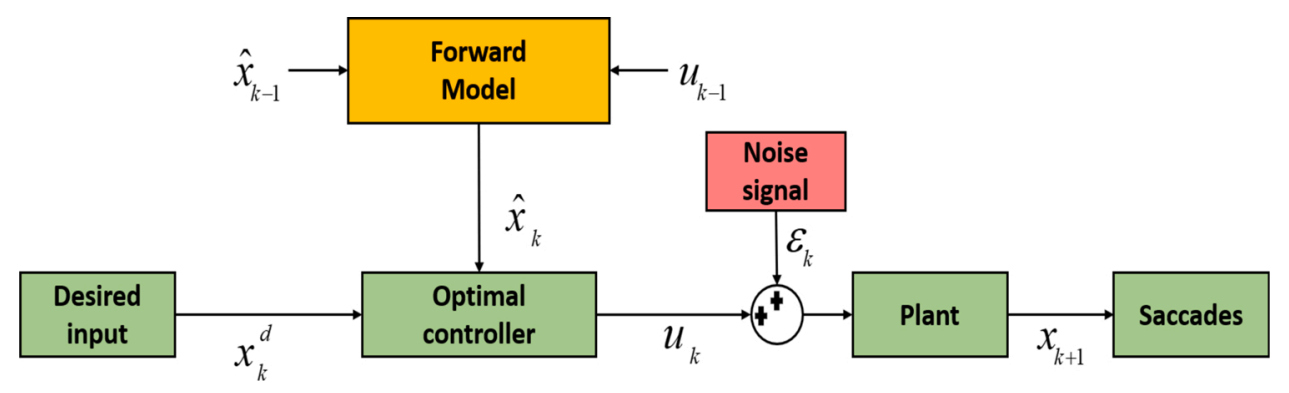

Fig. 1. Block diagram of velocity tracking model.

In (3), $\tau_{1}$ and $\tau_{2}$ are given by $\tau_{1}=B / K$ and $\tau_{2}=J / B$, with values assumed to be $223 \mathrm{~ms}$ and $14 \mathrm{~ms}$, respectively as in $[21,22]$. This system dynamics is valid only when considering horizontal saccades $\left(0^{\circ}\right.$ or $180^{\circ}$ directions).

For oblique saccades (see Section 3.1), the system dynamics involves a horizontal component and a vertical component separately. Hence, in the case of oblique saccades the state $x(t) \triangleq\left[\theta^{h}(t), \dot{\theta}^{h}(t), \theta^{v}(t), \dot{\theta}^{v}(t)\right]^{T}$ and control $u(t) \triangleq\left[u^{h}(t), u^{v}(t)\right]^{T}$. The state matrices $A_{c}$ and $B_{c}$ are defined as follows

$$
\begin{aligned}
& A_{c} \triangleq\left[\begin{array}{cccc}
0 & 1 & 0 & 0 \\
\frac{-1}{\tau_{1} \tau_{2}} & -\left(\frac{\tau_{1}+\tau_{2}}{\tau_{1} \tau_{2}}\right) & 0 & 0 \\
0 & 0 & 0 & 1 \\
0 & 0 & \frac{-1}{\tau_{1} \tau_{2}} & -\left(\frac{\tau_{1}+\tau_{2}}{\tau_{1} \tau_{2}}\right)
\end{array}\right] \\
& B_{c} \triangleq\left[\begin{array}{cccc}
0 & \frac{1}{\tau_{1} \tau_{2}} & 0 & 0 \\
0 & 0 & 0 & \frac{1}{\tau_{1} \tau_{2}}
\end{array}\right]^{T}
\end{aligned}
$$

This study has assumed that there is no coupling between the horizontal and vertical components in the oculomotor plant dynamics, similar to [17]. This makes the oblique saccade formulation equivalent to solving two one dimensional problems of horizontal component and vertical component of the saccade separately. In reality, it is possible that there is coupling between components in case of oblique saccades. A more nuanced model of oculomotor plant would be needed for studying these coupling effects.

Subsequently, discrete optimal control is used to solve this problem and hence there is a need for discretization of the state dynamics given in (2). The state dynamics in discrete form can be written for both the horizontal and oblique case as

$x_{k+1}=A x_{k}+B u_{k}$

with, $A=e^{A_{c} \Delta t}$ and $B=A_{c}^{-1}\left(e^{A_{c} \Delta t}-I\right) B_{c}$ representing the system matrices in discrete-time and $k$ denoting the discrete time-steps. A time step $\Delta t=4 \mathrm{~ms}$ is used for simulations, as decreasing the time steps further did not have any influence on the simulation results. Note that one can also use the first order approximation in discretization which gives $A=A_{c} \Delta t+I$ and $B=B_{c} \Delta t$. However, in both cases the results had no appreciable difference.

\subsection{Noise in the control signal}

Noise affects the saccadic system at different levels like target localization, movement planning, and execution [6]. Here, it is assumed that the effect of all these noises would be reflected as an uncertainty added to the control signal acting on the plant. Interestingly, it turns out that the noise affecting the neural command is signal-dependent, which means that the standard deviation of the noise distribution scales with the mean value of the signal and hence is dependent on the mean control signal itself [22]. Such a noise structure is motivated by previous literature that suggested that the signal-dependent noise in the control signal can capture saccade trajectories and the main sequence [11]. There is also experimental evidence that suggests that the source of the signal-dependent noise in the case of isometric force production can be attributed to central voluntary control, rather than peripheral muscle components [23]. This suggests that the noise is added to the control signal $u_{k}$ before acting on the plant and the noise influence matrix in the system dynamics can be taken to be the same as the control influence matrix. Based on these observations, the stochastic system dynamics can be written as

$x_{k+1}=\mathrm{Ax}_{k}+B\left(u_{k}+\epsilon_{k}\right), \quad k=1,2, \ldots, n$

where $n$ is the number of time-steps in the simulation and $\epsilon_{k}=\alpha u_{k} w_{k}$, where, $w_{k}$ represents a random variable drawn from zero-mean Gaussian distribution with a variance of one and $\alpha$ is the noise scaling factor in the signal-dependent noise definition [22]. Note that, $\alpha$ is a free parameter estimated from experimental data. This structure of the noise is very important in further analyses.

\subsection{Forward model}

An important component in the proposed model is the presence of an internal feedback loop, which uses a copy of the control signal to estimate the state with the help of a forward model block (see Fig. 1). In a generic case, the estimate of the state would be obtained by combining the prediction in the current step with sensory observations obtained from output feedback. But in the case of the saccadic system, the output feedback is not available and hence the estimate at the current step depends only on the estimate in the previous step. Thus, given the copy of the un-corrupted control signal $u_{k}$, the forward model estimates the states at the next instant of time, $\widehat{x}_{k+1}$ as follows

$\widehat{x}_{k+1}=A_{f} \widehat{x}_{k}+B_{f} u_{k}$

where $\widehat{x}_{k}=\mathbb{E}\left[x_{k}\right]$. The forward model is assumed to be not affected by noise and hence can perfectly predict the states of the system and it is taken that $A_{f}=A$ and $B_{f}=B$ respectively.

\subsection{Velocity tracking optimal controller}

To investigate if the saccadic system utilizes a velocity tracking strategy to control saccades, an optimal control policy that is based on a desired velocity signal has to be calculated. The controller needs to convert the desired signal $x^{d}$ and the estimate of the state $\hat{x}$ at each step into a motor command or control signal $u$. This transformation is represented by the optimal controller block in Fig. 1.

The cost function $V$ that is minimized during the movement (with $N$ 
steps) is taken as the expectation of sum of costs at all steps from 1 to $N$ and is given by

$V=\mathbb{E}\left[\sum_{i=1}^{N} \psi_{i}\right]$

where $\psi_{i}$ is the cost at each step $i$ and $\mathbb{E}$ represents expectation operator. The expectation is taken because the state dynamics is stochastic and only its expectation can be known deterministically in this problem. The cost function is subject to the stochastic system dynamics $x_{k+1}=\mathrm{Ax}_{k}+$ $B\left(u_{k}+\epsilon_{k}\right)$ as given by (6), with the state vector $x_{i}=\left[\theta_{i}, \dot{\theta}_{i}\right]^{T}$ consisting of angular displacement $\theta_{i}$ and angular velocity $\dot{\theta}_{i}$. The initial condition of the state is fixed and taken as the value of mean angular displacement $\theta_{1}^{\mu}$ and mean angular velocity $\dot{\theta}_{1}^{\mu}$ at the first time step in the experimental data. The final conditions of the states are free. There are no constraints involved in this problem. The cost at each step $i$ is a combination of two terms, of which the first term makes sure that the error between the desired system states and the achieved state is minimum and the second term minimizes the effort by penalizing the control signal magnitude. It is given by

$\psi_{i}=\left(x_{i}-x_{i}^{d}\right)^{T} Q_{i}\left(x_{i}-x_{i}^{d}\right)+u_{i}^{T} R_{i} u_{i}$,

where the desired state is $x_{i}^{d}=\left[\theta_{i}^{d}, \dot{\theta}_{i}^{d}\right]^{T}$. The desired values of the state are modeled as the mean of the experimental data obtained for each subject across the trials. The error weightage matrix $Q_{i}$ and the control weightage matrix $R_{i}$ decides the distribution of the cost between the two terms. In this problem, $Q_{i}=[0,0 ; 0, q]$ where $q$ is a free parameter. The structure of $Q_{i}$ was chosen to have weightage to only velocity error as only desired velocity was available at the input. The control weighting parameter $R_{i}$ is fixed to one. It is observed that this assumption did not affect the solution since this is equivalent to redefining original error weightage with a scaling factor of $1 / R_{i}$ and the free parameter $q$ in the error weightage matrix absorbed this scaling effect.

\subsection{Control solution using dynamic programming approach}

The optimal control problem is solved using dynamic programming approach $[16,17]$. The cost from any time step $k$ can be written based on (8) as sum of costs from step $k$ to final step $N$ and is given by given by

$V_{k}^{*}\left(x_{k}, \widehat{x}_{k}\right)=\min _{u_{k}}\left\{\mathbb{E}\left[\psi_{k}\right]+\mathbb{E}\left[V_{k+1}^{*}\left(x_{k+1}, \widehat{x}_{k+1}\right) \mid x_{k}, \widehat{x}_{k}, u_{k}\right]\right\}$

For this problem considering cost per step as given in (12), the relationship in (13) becomes

$V_{k}^{*}\left(x_{k}, \widehat{x}_{k}\right)=\min _{u_{k}}\left\{\begin{array}{c}\left(x_{k}-x_{k}^{d}\right)^{T} Q_{k}\left(x_{k}-x_{k}^{d}\right)+u_{k}^{T} R_{k} u_{k} \\ +\mathbb{E}\left[V_{k+1}^{*}\left(x_{k+1}, \widehat{x}_{k+1} \mid x_{k}, \widehat{x}_{k}, u_{k}^{*}\right)\right]\end{array}\right\}$

The optimal control $u_{k}^{*}$ can be obtained by solving (14) by using a value approximation method as described in $[15,25]$. Given a problem with value function dependent on the state $x_{k}$ and the estimate of the state $\widehat{x}_{k}$, the approximate form of value under optimal control policy for achieving tracking of a desired state can be written as

$V_{k}=x_{k}^{T} W_{k}^{x} x_{k}-2 x_{k}^{T} W_{k}^{r}+e_{k}^{T} W_{k}^{e} e_{k}+W_{k}$

where $e_{k} \triangleq x_{k}-\widehat{x}_{k}$ and $W_{k}^{x}, W_{k}^{e}, W_{k}^{r}$ and $W_{k}$ are the weightages. The variable $e_{k}$ is the estimation error between the current state of the system and the estimated state predicted by the forward model. The right hand side of expression in (14) is expanded to get

$$
\begin{aligned}
V_{k}= & \left(x_{k}-x_{k}^{d}\right)^{T} Q_{k}\left(x_{k}-x_{k}^{d}\right)+u_{k}^{T} R_{k} u_{k}+\mathbb{E}\left[x_{k+1}^{T} W_{k+1}^{x} x_{k+1}+e_{k+1}^{T} W_{k+1}^{e} e_{k+1}\right. \\
& \left.-2 x_{k+1}^{T} W_{k+1}^{r}+W_{k+1}\right]
\end{aligned}
$$

by using the approximated form of value in (15). It is known that for any random variable $z_{k}, \mathbb{E}\left[z_{k}^{T} \mathrm{~A} z_{k}\right]$ can be expanded in terms of expectation (E) and variance (Var) of $z_{k}$ as

$\mathbb{E}\left[z_{k}^{T} A z_{k}\right]=\mathbb{E}\left[z_{k}\right]^{T} A \mathbb{E}\left[z_{k}\right]+\operatorname{Tr}\left[A\left\{\operatorname{Var}\left[z_{k}\right]\right\}\right]$,

where $\operatorname{Tr}$ represents the trace operator. Using this property, the quadratic term in $x_{k+1}$ in (16) can be expanded as follows

$\mathbb{E}\left[x_{k+1}^{T} W_{k+1}^{x} x_{k+1}\right]=\mathbb{E}\left[x_{k+1}\right]^{T} W_{k+1}^{x} \mathbb{E}\left[x_{k+1}\right]+\operatorname{Tr}\left[W_{k+1}^{x} \operatorname{Var}\left[x_{k+1}\right]\right]$

Further, $\mathbb{E}\left[x_{k+1}\right]$ and $\operatorname{Var}\left[x_{k+1}\right]$ is got from the state dynamics equation given by (6) and the following expression is obtained

$\mathbb{E}\left[x_{k+1}^{T} W_{k+1}^{x} x_{k+1}\right]=\left(A x_{k}+B u_{k}\right)^{T} W_{k+1}^{x}\left(A x_{k}+B u_{k}\right)+\operatorname{Tr}\left[\alpha u_{k} B^{T} W_{k+1}^{x} B u_{k} \alpha\right]$

$\mathbb{E}\left[e_{k+1}^{T} W_{k+1}^{e} e_{k+1}\right]=\mathbb{E}\left[x_{k+1}-\widehat{x}_{k+1}\right]^{T} W_{k+1}^{e} \mathbb{E}\left[x_{k+1}-\widehat{x}_{k+1}\right]+\operatorname{Tr}\left[W_{k+1}^{e} \operatorname{Var}\left[x_{k+1}-\widehat{x}_{k+1}\right]\right]$

$V_{k}=\mathbb{E}\left[\sum_{i=k}^{N} \psi_{i}\right]$,

which can be then rewritten as

$V_{k}=\mathbb{E}\left[\psi_{k}+V_{k+1}\right]=\mathbb{E}\left[\psi_{k}\right]+\mathbb{E}\left[V_{k+1}\right]$,

where $\psi_{k}$ is the cost at step $k$ and $V_{k+1}$ is cost-to-go from time $k+1$ to $N$. The term $\psi_{k}$ is defined based on (9) as

$\psi_{k}=\left(x_{k}-x_{k}^{d}\right)^{T} Q_{k}\left(x_{k}-x_{k}^{d}\right)+u_{k}^{T} R_{k} u_{k}$

If the optimal control, state and cost for all values from $k+1$ to $N$ are found, then the optimal value for the step $k$ to $k+1$ can be found out using the functional equation of dynamic programming [24], which is
The same property as given by (17) is used to expand the expectation of the quadratic term in $e_{k+1}$ in (16) and the expression is obtained as

Using the state dynamics given by (6) and forward model dynamics given by (7) this expression can be written as

$\mathbb{E}\left[e_{k+1}^{T} W_{k+1}^{e} e_{k+1}\right]=e_{k}^{T} A^{T} W_{k+1}^{e} A e_{k}+\operatorname{Tr}\left[\alpha u_{k} B^{T} W_{k+1}^{e} B u_{k} \alpha\right]$

Also, $\mathbb{E}\left[x_{k+1}^{T} W_{k+1}^{r}\right]$ can be written as

$\mathbb{E}\left[x_{k+1}^{T} W_{k+1}^{r}\right]=\left(W_{k+1}^{r}\right)^{T} \mathbb{E}\left[x_{k+1}\right]=\left(W_{k+1}^{r}\right)^{T}\left(A x_{k}+B u_{k}\right)$

Thus, the value function in (16) can be expanded using (18), (19) and (20) to get 


$$
\begin{aligned}
V_{k}= & \left(x_{k}-x_{k}^{d}\right)^{T} Q_{k}\left(x_{k}-x_{k}^{d}\right)+u_{k}^{T} R_{k} u_{k}+\left(A x_{k}+B u_{k}\right)^{T} W_{k+1}^{x}\left(A x_{k}+B u_{k}\right) \\
& +\operatorname{Tr}\left[\alpha u_{k} B^{T} W_{k+1}^{x} B u_{k} \alpha\right]+e_{k}^{T} A^{T} W_{k+1}^{e} A e_{k}+\operatorname{Tr}\left[c u_{k} B^{T} W_{k+1}^{e} B u_{k} c\right] \\
& -2\left(W_{k+1}^{r}\right)^{T}\left(A x_{k}+B u_{k}\right)+W_{k+1}
\end{aligned}
$$

Applying the necessary condition of optimality given by

$\left.\frac{\partial V_{k}}{\partial u_{k}}\right|_{u=u_{k}^{*}}=0$

with $V_{k}$ as in (21), the optimal control signal $u_{k}^{*}$ at step $k$ can be obtained. Eq. (22) leads to the following expression

$$
\begin{aligned}
W_{k}^{x} & =Q_{k}+A^{T} W_{k+1}^{x} A-A^{T} W_{k+1}^{x} B L^{-1} B^{T} W_{k+1}^{x} A \\
W_{k}^{e} & =A^{T} W_{k+1}^{e} A+A^{T} W_{k+1}^{x} B L_{k}{ }^{-1} B^{T} W_{k+1}^{x} A \\
W_{k}^{r} & =A W_{k+1}^{r}+Q_{k} x_{k}^{d}-A^{T} W_{k+1}^{x} B L_{k}^{-1} W_{k+1}^{r} B \\
W_{k} & =W_{k+1}+2 x_{k}^{d^{T}} Q_{x} x_{k}^{d}-B^{T} W_{k+1}^{r} L_{k}^{-1} W_{k+1}^{r}{ }^{T} B
\end{aligned}
$$

Note that in (28), $G_{k}$ and $b_{k}$ are written in terms of their expansions given by (25) and (27), respectively. The weight matrices at final step $N$ can be obtained as $W_{N}^{x}=Q_{N}, W_{N}^{r}=Q_{N} x_{N}^{d}, W_{N}^{e}=0$ and $W_{N}=$ $2 x_{N}^{d}{ }^{T} Q_{N} x_{N}^{d}$, by substituting all weights in step $N+1$ as zero in (28). Subsequently, these weights are used in (28) to calculate the weights in the second last step $N-1$ and recursively backward till first step. Using these weights, the feedback control gain $G_{k}$ and the bias $b_{k}$ are calculated

$R_{k} u_{k}^{*}+B^{T} W_{k+1}^{x} B u_{k}^{*}+\operatorname{Tr}\left[\alpha B^{T} W_{k+1}^{x} B \alpha\right] u_{k}^{*}+\operatorname{Tr}\left[\alpha B^{T} W_{k+1}^{e} B \alpha\right] u_{k}^{*}+B^{T} W_{k+1}^{x} A x_{k}-\left(W_{k+1}^{r}\right)^{T} B=0$

which is grouped and rearranged to obtain the optimal control $u_{k}^{*}$ as

$u_{k}^{*}=-G_{k} x_{k}+b_{k}$

with the feedback gain given by

$G_{k}=L_{k}^{-1} B^{T} W_{k+1}^{x} A$

where,

$L_{k} \triangleq R_{k}+C_{k+1}^{x}+C_{k+1}^{e}+B^{T} W_{k+1}^{x} B$

with $C_{k+1}^{x}$ and $C_{k+1}^{e}$ defined as $C_{k+1}^{x} \triangleq \operatorname{Tr}\left(\alpha B^{T} W_{k+1}^{x} B \alpha\right)$ and $C_{k+1}^{e} \triangleq$ $\operatorname{Tr}\left(\alpha B^{T} W_{k+1}^{e} B \alpha\right)$ respectively and the bias term given by

$b_{k} \triangleq L_{k}^{-1}\left(W_{k+1}^{r}\right)^{T} B$

Since there is no external state feedback information, the forward model is used to estimate the expectation of the current state of the system as in (7), and the control signal is calculated on this estimated state as $u_{k}^{*}=-$ backward from the last time step using the (25) and (27) respectively. The gains $G_{k}$ and $b_{k}$ are used to calculate the optimal control signal for each step using $-G_{k} \widehat{x}_{k}+b_{k}$, while simultaneously propagating the forward model to get an estimate of the state $\widehat{x}_{k}$.

\subsection{Verification of optimality of value function}

The optimal control signal obtained by solving the necessary conditions of optimality is in terms of the actual state $x_{k}$. However, since the state feedback is not available, the estimate of the state $\widehat{x}_{k}$ given by the forward model is used for calculating the optimal control. This necessitates to check whether the new optimal value function obtained based on this optimal control calculated from $-G_{k} \widehat{x}_{k}+b_{k}$ will be of the same form as assumed in (15).

To carry out this check, the optimal control expression with $x_{k}$ replaced by $\widehat{x}_{k}$ is substituted in (21) and the optimal value function is obtained. The optimal value after regrouping the terms quadratic in $u_{k}^{*}$ is given by,

$$
\begin{gathered}
V_{k}=\left(x_{k}-x_{k}^{d}\right)^{T} Q_{k}\left(x_{k}-x_{k}^{d}\right)+\left\{\left(-G_{k} \widehat{x}_{k}+b_{k}\right)^{T}\left(R_{k}+B^{T} W_{k+1}^{x} B+C_{k+1}^{x}+C_{k+1}^{e}\right)\right. \\
\left.\left(-G_{k} \widehat{x}_{k}+b_{k}\right)\right\}+\left(A x_{k}\right)^{T} W_{k+1}^{x}\left(A x_{k}\right)+e_{k}^{T} A^{T} W_{k+1}^{e} A e_{k} \\
-2 W_{k+1}^{r}{ }^{T}\left(A x_{k}+B u_{k}^{*}\right)+W_{k+1}
\end{gathered}
$$

$G_{k} \widehat{x}_{k}+b_{k}$. In the next section (Section 2.6), it is shown that in spite of calculating optimal control signal from estimate of the state, the corresponding optimal value function obtained takes the same optimal form as assumed in (15).

The update equations for the weight matrices $W_{k}^{x}, W_{k}^{e}, W_{k}^{r}$ and $W_{k}$ can be obtained by substituting the optimal control expression $-G_{k} \widehat{x}_{k}+b_{k}$ in the optimal value function obtained in (21). After simplification, it is observed that the the left out terms can be regrouped into terms quadratic in $x_{k}$, quadratic in $e_{k}$, linear in $x_{k}$ and constant term. By comparing these terms with similar terms in approximate form of value function given in (15), the weight update equations are obtained. The update equations turn out to be backward recursive equations dependent on system matrices $A$ and $B$, the feedback gain $G_{k}$ and the bias $b_{k}$. They are given by
This function is expanded using (25) and (27) and obtained as

$$
\begin{aligned}
V_{k}= & x_{k}^{T} Q_{k} x_{k}+x_{k}^{d^{T}} Q_{k} x_{k}^{d}-2 x_{k}^{T} Q_{k} x_{k}^{d}+\widehat{x}_{k}^{T} Z \widehat{x}_{k}-2 B^{T} W_{k+1}^{x} A \widehat{x}_{k} L_{k}^{-1} W_{k+1}^{r}{ }^{T} B \\
& +B^{T} W_{k+1}^{r} L_{k}{ }^{-1} W_{k+1}^{r} B+x_{k}^{T} A^{T} W_{k+1}^{x} A x_{k}-2 \widehat{x}_{k}{ }^{T} Z x_{k} \\
& +2 x_{k}^{T} A^{T} W_{k+1}^{x} B L_{k}^{-1} W_{k+1}^{r} B+e_{k}^{T} A^{T} W_{k+1}^{e} A e_{k}-2 W_{k+1}^{r}{ }^{T} A x_{k} \\
& +2 W_{k+1}^{r} B L_{k}^{-1} B^{T} W_{k+1}^{x} A \widehat{x}_{k}-2 B^{T} W_{k+1}^{r} L_{k}^{-1} W_{k+1}^{r} B+W_{k+1}
\end{aligned}
$$

The expansion consists of terms which are quadratic and linear in $x_{k}$, quadratic in $e_{k}$, quadratic and linear in $\hat{x}_{k}$ as well as constants. In (30), the fifth term and twelfth term cancel each other. The terms in $\widehat{x}_{k}$ can be eliminated by replacing the fourth term and eighth term together using the expression 
$\widehat{x}_{k}^{T} Z \widehat{x}_{k}-2 \widehat{x}_{k}^{T} Z_{x_{k}}=\left(x_{k}-\widehat{x}_{k}\right)^{T} Z\left(x_{k}-\widehat{x}_{k}\right)-x_{k}^{T} \mathrm{Zx}_{k}$

where $Z=A^{T} W_{k+1}^{x} \mathrm{BL}_{k}^{-1} B^{T} W_{k+1}^{x} A$ and the first term is quadratic in $e_{k}$ while the second term is quadratic in $x_{k}$. The reduced optimal value function equation then becomes

$$
\begin{aligned}
V_{k}= & x_{k}^{T}\left(Q_{k}+A^{T} W_{k+1}^{x} A-Z\right) x_{k}+e_{k}^{T}\left(A^{T} W_{k+1}^{e} A+Z\right) e_{k}-2 x_{k}^{T}\left(W_{k+1}^{r}{ }^{T} A\right. \\
& \left.+Q_{k} x_{k}^{d}-A^{T} W_{k+1}^{x} B L_{k}^{-1} W_{k+1}^{r} B\right)-B^{T} W_{k+1}^{r}{ }^{T} L_{k}^{-1} W_{k+1}^{r} B+x_{k}^{d^{T}} Q_{k} x_{k}^{d}+W_{k+1}
\end{aligned}
$$

It is clear from (32) that even though a modified optimal control signal based on the estimate of the state is used in place of the actual expression obtained in (24), the optimal value function remains of the same optimal form which was assumed in (15). Hence, the assumption of using estimated of the state in place of actual state is justified and the approximated value of the form given in (15) can be used.

In this section, a desired velocity tracking optimal controller which converts the estimate of the actual state (which is given by the forward model in the internal feedback loop) to produce the control signals that would serve as motor commands to the oculomotor plant is obtained. The mean trajectories of displacement and velocity are simulated by substituting optimal control signal in the expectation of the state dynamics equation given by (6). To verify the proposed model of saccade control based on velocity tracking, the predictions of this saccade generation framework are tested using behavioral data of saccades obtained from the human experiment. The details of the experiment are discussed in Section 3.

\subsection{Model parameter estimation}

The model has two free parameters, $q$ and $c$, which are tuned individually for each subject. The model parameters were estimated by a two-stage process. The parameter $q$ in the error weightage matrix in the cost function (refer Section 2.4) was estimated such that the error between the velocity prediction of the model and experimental velocity was minimized. This was done after setting the noise scaling parameter $\alpha$ to zero. The error is defined as

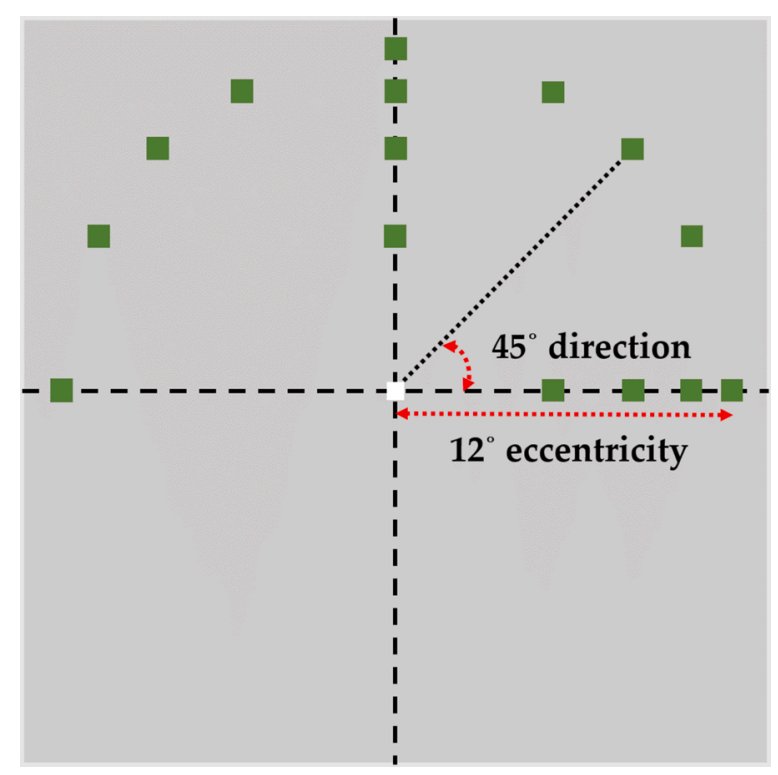

Fig. 2. Task paradigm showing all stimulus positions.

$$
e=\frac{\sqrt{\sum_{j=1}^{m}\left[z_{\mu}^{\text {expt }}\left(t_{j}\right)-z_{\mu}^{\text {pred }}\left(t_{j}\right)\right]^{2}}}{\sqrt{\sum_{j=1}^{m}\left[z_{\mu}^{\text {expt }}\left(t_{j}\right)\right]^{2}}}
$$

where $z$ is defined as the variable of interest. It was taken as the angular velocity $(\dot{\theta})$ for obtaining the free parameters. Here, $\dot{\theta}_{\mu}^{\text {expt }}$ is defined as the mean angular velocity obtained for individual subjects based on experimental data and $\dot{\theta}_{\mu}^{\text {pred }}$ is the model's prediction of the same. The second stage in the estimation process was of tuning the noise scaling parameter $\alpha$ (refer Section 2.2). This parameter was obtained by minimizing the same error as described in (33), but with the value of $q$ in the model fixed at the estimated value. The parameter ranges and guess values for the optimization are obtained by scanning the parameter space. The parameters were estimated using saccades made to horizontal leftward target at $12^{\circ}$ eccentricity in $180^{\circ}$ direction (refer to Section 3 ) and were used for all further predictions of the model. The errors in fit were also quantified using (33) by defining the variable $z$ as $\theta$ or $\dot{\theta}$ depending on whether fit error in displacement was quantified or fit error in velocity, respectively. The errors are all expressed in percentages.

\section{Experimental Data}

The behavioral data were collected from 20 healthy subjects in the age group of 20-33 years in a laboratory set-up. Each of the 20 participants carried out repeated trials of saccades to the same targets during the experiment. The participants consisted of an equal number of men and women. The experimental study was approved by the Institute Human Ethics Committee of the Indian Institute of Science (IHEC No: 128102016 approved on 28-10-2016). Informed consent was obtained from all participants in accordance with the guidelines. Participants were monetarily compensated for taking part in the experiment.

\subsection{Experiment setup and design}

Saccadic eye movement data used in this study was collected using an IR based pupil tracking camera (ISCAN, Boston, USA) at a sampling rate of $240 \mathrm{~Hz}$. Each participant was made to sit in front of the LCD

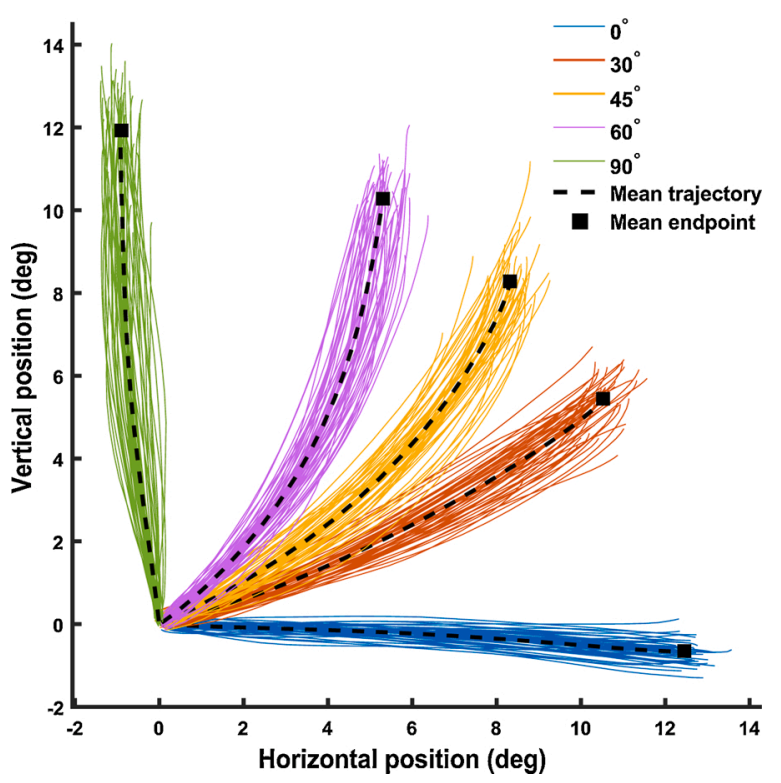

Fig. 3. Example of saccades made to different target directions by a subject. 


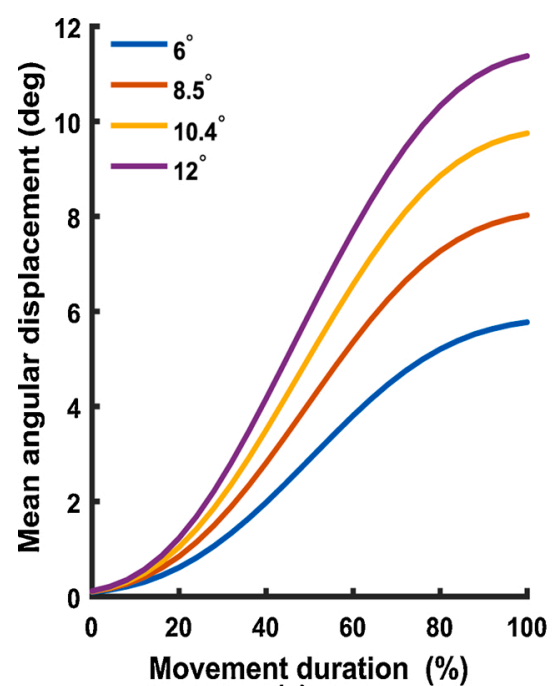

(a)

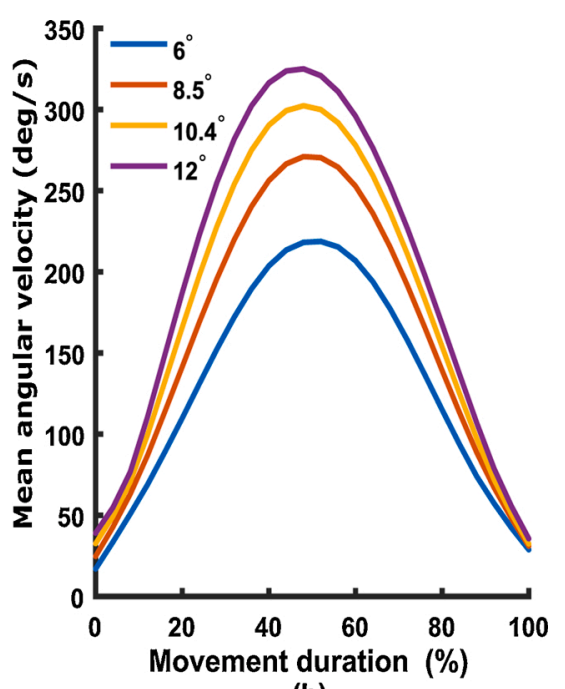

(b)

Fig. 4. Mean angular displacement and velocity profiles of saccades to different target amplitudes for an example subject.

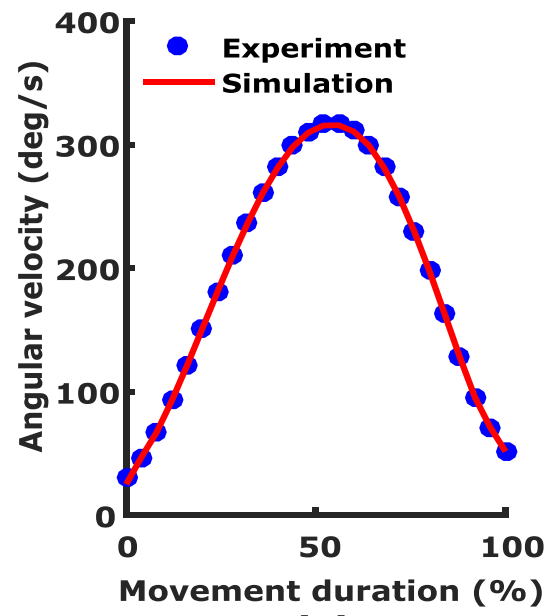

(a)

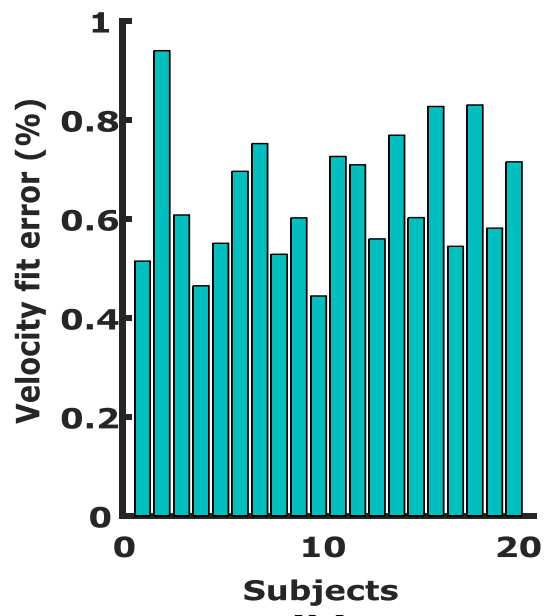

(b)

Fig. 5. Angular velocity fit of the proposed model.

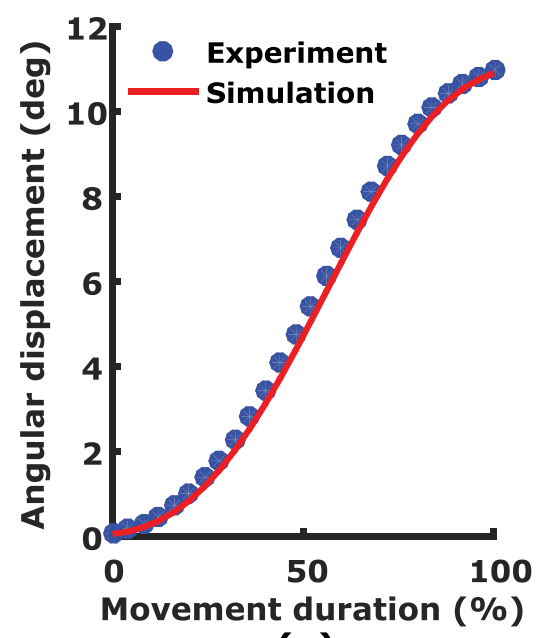

(a)

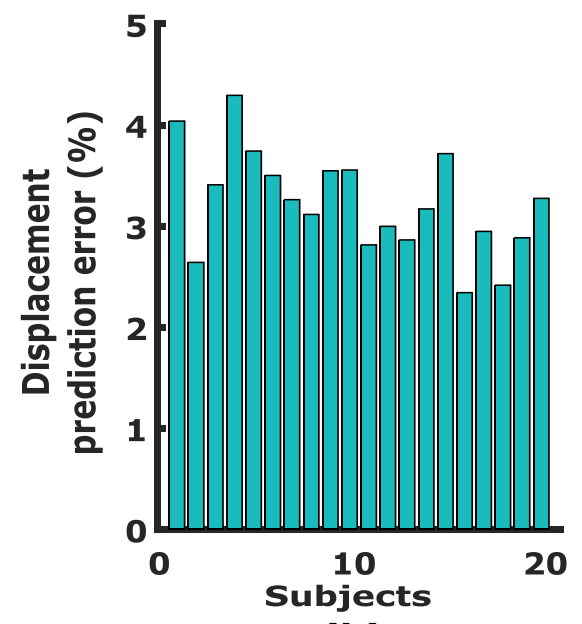

(b)

Fig. 6. Model prediction of mean angular displacement of saccades for $12^{\circ}$ horizontal leftward saccades. 
screen on which stimuli were shown. They were also made to rest their chin such that the eye-level matched the center of the screen. At the same time, the head was locked at the temple to obtain eye movements exclusively in the absence of any head movements.

The experiment had 15 different targets shown on the upper half of the screen in a randomized fashion as shown in Fig. 2. It included targets in 9 different directions including $0^{\circ}, 30^{\circ}, 45^{\circ}, 60^{\circ}, 90^{\circ}, 120^{\circ}, 135^{\circ}, 150^{\circ}$ and $180^{\circ}$ at an eccentricity of $12^{\circ}$ as well as targets at three different eccentricities of $6^{\circ}, 8.5^{\circ}$ and $10.4^{\circ}$ in both $0^{\circ}$ and $90^{\circ}$ directions. Each trial started with a white fixation point shown in the middle of the screen. After a $400 \pm 60 \mathrm{~ms}$ delay, the fixation box disappeared and a green target appeared at the periphery. The subject was instructed to make one single saccadic eye movement to the target as soon as it appeared. A green tick mark on the screen and a beep sound gave feedback to the subject about the success of the trial.

\subsection{Data analysis}

Saccade displacement was calculated from horizontal and vertical positions obtained from the eye camera. It was fit using a polynomial function of seventh order and differentiated to obtain the velocity profiles. The saccade start and end were detected based on a velocity criterion. The time at which the velocity of the eye exceeds $10 \%$ of the peak velocity was marked as the start of the saccade and the time point where the velocity reduces below the same threshold after the peak velocity was considered the end of the saccade. Since each trial produced saccades of different duration, the time was normalized into equal bins for the entire saccade duration. This allowed the calculation of the mean saccade trajectory across trials at these bins.

\section{Results Analysis}

An example of saccade trajectories made by a subject across multiple trials for different oblique targets is shown in Fig. 3. Further analyses were carried out on the mean of the trajectories calculated across trials, which is shown with black dashed lines in the figure.

The mean angular displacement and angular velocity for an example subject who participated in the experiment are shown in Fig. 4 for four different targets amplitudes of $6^{\circ}, 8.5^{\circ}, 10.4^{\circ}$ and $12^{\circ}$. The mean angular displacement is typically a monotonously increasing function of time while the mean angular velocity peaks towards the middle of the movement and then decreases. Further, saccadic eye movements with

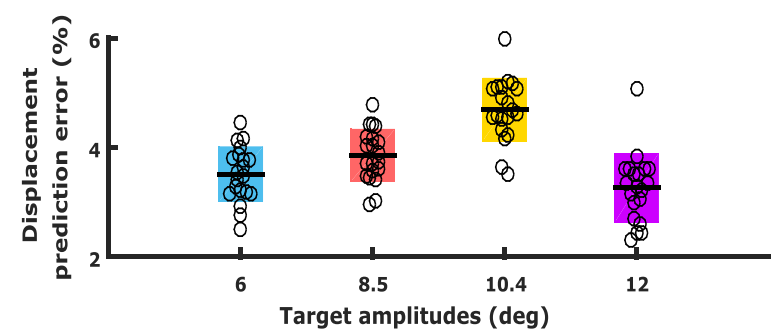

(a)

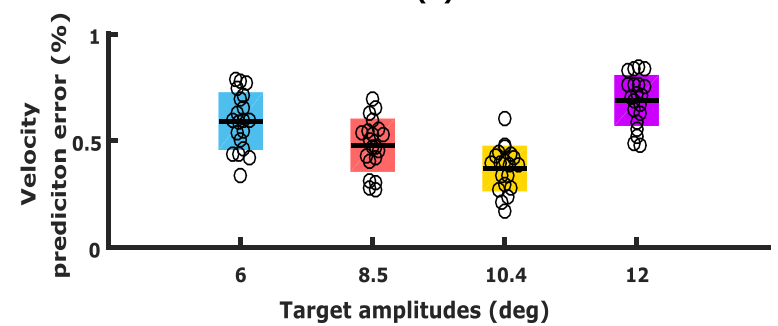

(b)

Fig. 7. Model prediction errors in angular displacement and velocity for saccades to different target amplitudes.
Table 1

Model prediction errors across amplitudes.

\begin{tabular}{lll}
\hline \multirow{2}{*}{ Target amplitude } & \multicolumn{2}{l}{ Prediction error (mean $\left.\pm \mathrm{std}^{\mathrm{a}} \%\right)$} \\
\cline { 2 - 3 } & Displacement & Velocity \\
\hline $6^{\circ}$ & $3.51 \pm 0.49$ & $0.59 \pm 0.13$ \\
$8.5^{\circ}$ & $3.85 \pm 0.47$ & $0.47 \pm 0.12$ \\
$10.4^{\circ}$ & $4.69 \pm 0.56$ & $0.37 \pm 0.10$ \\
$12^{\circ}$ & $3.26 \pm 0.62$ & $0.68 \pm 0.11$ \\
\hline
\end{tabular}

a standard deviation.

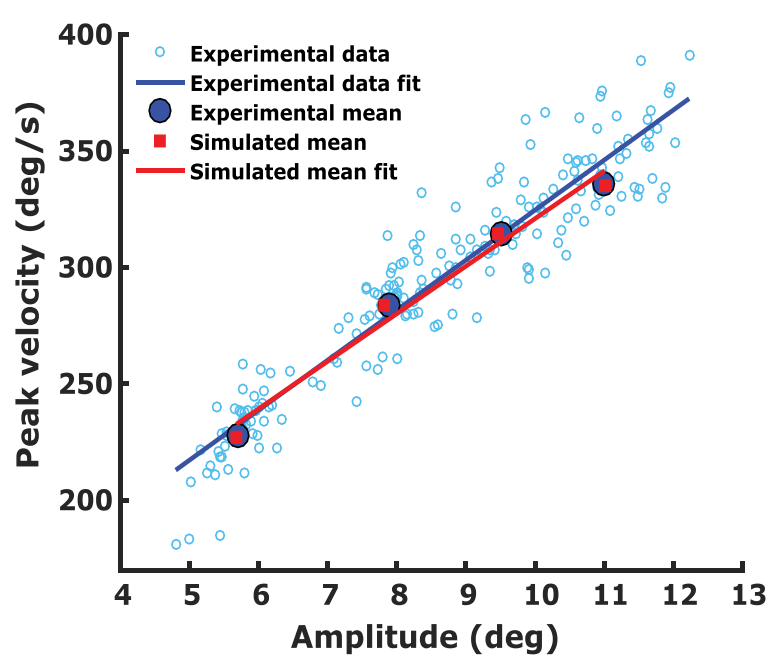

(a)

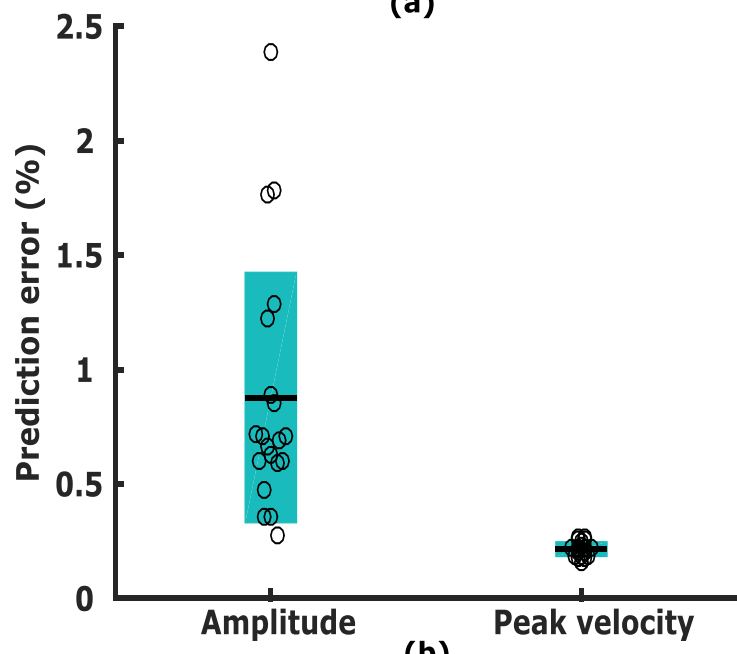

(b)

Fig. 8. Model prediction of main sequence relationship between saccade amplitude and peak velocity.

larger amplitudes have higher peak velocities as is observed in general $[26,11]$.

The proposed model's free parameters were estimated from $12^{\circ}$ horizontal leftward saccades as described in Section 2.7 using the experimental mean angular velocity. An example fit for an individual subject is shown in Fig. 5(a). The errors in the fit as given by (33) and is shown for all the 20 subjects in Fig. 5(b). The mean error in velocity fit was $0.65 \%$ and the standard deviation was $0.13 \%$. The fit errors for all subjects were less than $1 \%$.

The predictions of mean angular displacement for the same has been presented separately to validate the model. The prediction error was also calculated and expressed in \% by using (33) as described in Section 2.7. Fig. 6(a) shows an example of the prediction of mean angular 


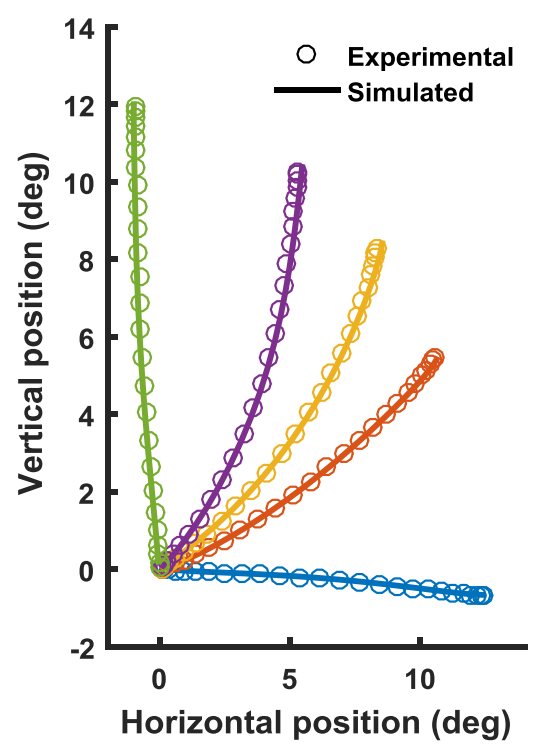

(a)

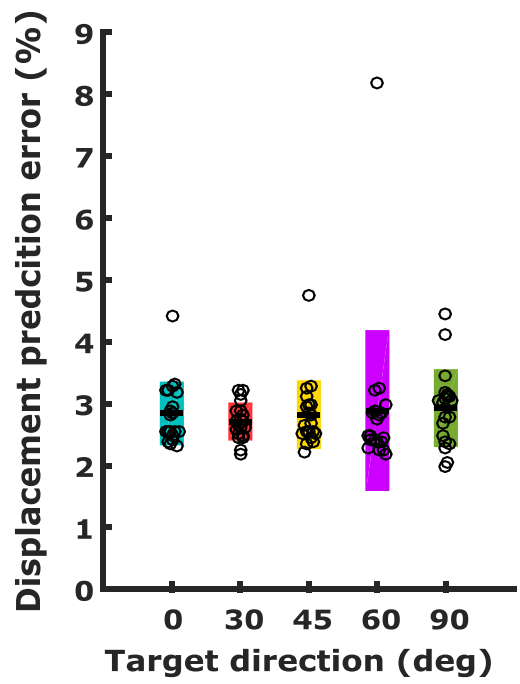

(c)

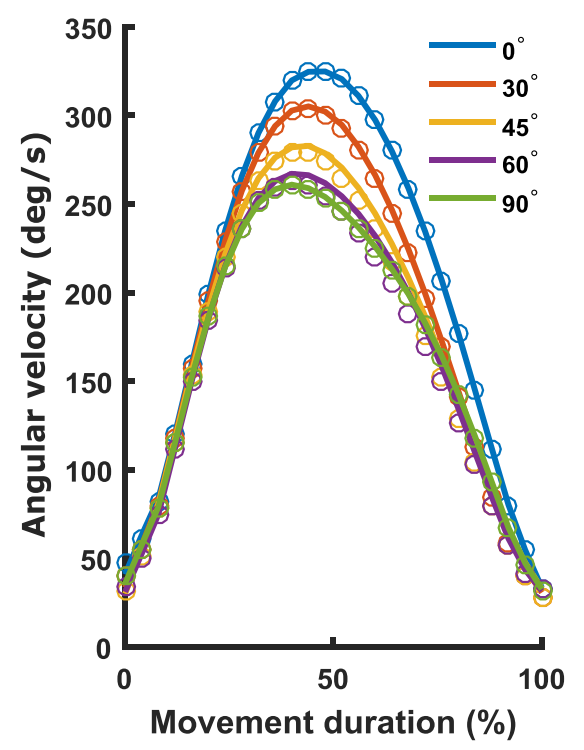

(b)

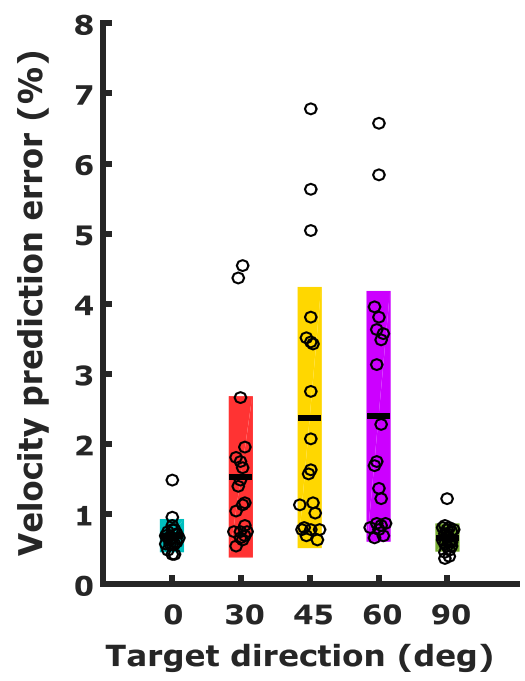

(d)

Fig. 9. Model prediction of saccade trajectory for oblique saccades and error quantification.

displacement for the same $12^{\circ}$ horizontal leftward saccades which is used for fitting the parameters. The prediction errors quantified for all 20 subjects are shown in Fig. 6(b). All prediction errors of the model were less than $5 \%$. The mean error in prediction of angular displacement, in this case, was $3.2 \%$ with a standard deviation of $0.5 \%$. Thus, the model can capture the saccade kinematics with low errors.

Table 2

Model prediction errors across directions.

\begin{tabular}{lll}
\hline \multirow{2}{*}{ Target direction } & \multicolumn{2}{l}{ Prediction error $\left(\right.$ mean $\left.\pm \mathrm{std}^{\mathrm{a}} \%\right)$} \\
\cline { 2 - 3 } & Displacement & Velocity \\
\hline $0^{\circ}$ & $2.84 \pm 0.50$ & $0.69 \pm 0.22$ \\
$30^{\circ}$ & $2.71 \pm 0.29$ & $1.53 \pm 1.14$ \\
$45^{\circ}$ & $2.82 \pm 0.54$ & $2.37 \pm 1.85$ \\
$60^{\circ}$ & $2.88 \pm 1.28$ & $2.39 \pm 1.77$ \\
$90^{\circ}$ & $2.92 \pm 0.62$ & $0.66 \pm 0.19$ \\
\hline
\end{tabular}

a standard deviation.
To check the generalizability of the model to other amplitudes, the model predictions of mean angular displacement and velocity of saccades made to the horizontal rightward targets at $6^{\circ}, 8.5^{\circ}, 10.4^{\circ}$ and $12^{\circ}$ were simulated. The free parameters were set to the already estimated values from $12^{\circ}$ horizontal leftward saccades. The errors in prediction for all amplitudes across 20 subjects are quantified in Fig. 7 for each of the amplitudes. Fig. 7(a) shows the prediction errors for angular displacement while that in Fig. 7(b) shows the prediction errors for angular velocity. Displacement prediction errors across subjects for all amplitudes were less than $6 \%$, while errors for velocity were all less than $1 \%$. In general, the errors in the prediction of angular velocity were much lesser compared to the prediction error in angular displacement for all target amplitudes. This is expected given that the model is tracking the desired velocity. The mean and standard deviation of the prediction errors across subjects are presented in Table 1.

Further validation of the model was also carried out by verifying the model's ability to predict the main sequence relationship between saccade amplitude and peak velocity (Fig. 8). The close correspondence between the fit and the data indicated that the model can capture the 
main sequence relationship between saccade amplitude and peak velocity (see example in Fig. 8(a)). To quantify the same across subjects, the prediction errors in mean saccade amplitude and mean saccade peak velocity between the experimental data and model simulation were quantified and are shown in Fig. 8(b). The errors were all less than 2.5\%. However, the non-linearity of this relationship at higher amplitudes could not be tested as all the amplitudes available in the experimental data were below $12^{\circ}$, which was in the linear part of the main sequence.

All the results presented until now were based on horizontal saccades. Hence, to further test the capability of the proposed velocity tracking model, simulations of saccades made to different oblique and vertical directions were carried out. For this purpose, the modified model with system dynamics with system matrices as mentioned in (4). It may be noted here that any possible interactions that may be present between the horizontal and vertical components in case of oblique saccades have not been considered in this work and may be considered a shortcoming of our study. The model with modified system dynamics was used to generate saccades made to $12^{\circ}$ eccentricity in oblique directions of $30^{\circ}, 45^{\circ}, 60^{\circ}$ and vertical direction of $90^{\circ}$, relative to the horizontal axis. The same parameters estimated with $12^{\circ}$ leftward saccades were used. An example of the model's prediction for one subject for position and velocity are shown in Fig. 9(a) and (b), respectively. The $0^{\circ}$ target is the same as horizontal $12^{\circ}$ in the across target amplitude analysis and is presented again for completion. The quantification of the error in prediction for all 20 subjects is shown for all directions in Fig. 9 (c) for displacement and in Fig. 9(d) for angular velocity. The mean and standard deviation in these predictions for oblique and vertical saccades are given in Table 2 .

Although the prediction error in the case of oblique saccades is generally higher than those of the horizontal saccades especially in case of velocity, the predictions were still reasonably good. The errors on an average were $2.84 \pm 0.65 \%$ for angular displacement and $1.53 \pm 1.03 \%$ for angular velocity. This may be because, unlike horizontal saccades, oblique and vertical saccades involve more oculomotor muscles and there might be some interactions, at the oculomotor plant or before, which are not being considered in this work. Another possible reason could be that the parameters fixed based on horizontal saccades may not be optimal for the oblique and vertical saccades.

\section{Conclusion and Discussions}

A novel velocity tracking optimal control model has been developed for saccade generation. The velocity tracking controller computes the optimal control signal based on an instantaneous error calculated using the internal feedback information. The model proposes a control mechanism in the presence of signal-dependent noise. The proposed model was tested using experimental data of saccade behavior collected from human participants. The model predicted the angular displacement profiles of horizontal saccades made to different amplitudes with high accuracy. It could also capture the main sequence relationship between saccade amplitude and peak velocity in the linear range. The model was also capable of predicting oblique saccades and vertical saccades made to targets in different directions. This is the first-ever model to show how the saccadic control system could use a desired velocity signal at its input for producing accurate saccades. The control algorithm proposed is also new as there are no tracking optimal control formulations with internal feedback philosophy available in the literature. Although this work demonstrates the application of the algorithm for the saccadic system, the framework may be utilized for gaining deeper insights regarding similar systems with internal feedback and tracking control.

The predominantly popular endpoint-based saccade control models were motivated by neurophysiological studies about the presence of a spatial map in the midbrain superior colliculus (SC), which is thought to be the center providing the desired goal to the brainstem saccade generation circuit (for instance see $[27,28,11,17]$ ). However, and interestingly, reversible inactivation of SC has shown that the trajectory of the saccade itself is altered, apart from the amplitude and direction of the saccades [29]. There are also a few studies that have shown the average firing rates in collicular cells to be correlated with peak saccadic velocity $[30,31]$. However, since saccades show a tight nonlinear coupling between amplitudes and peak velocities, the saccade generation system cannot achieve a particular saccade amplitude based on just the peak velocity information. Rather, the SC neurons would require to encode the entire velocity profile, as its integral would give the amplitude. This hypothesis was indeed shown to be true by the latest findings from a neurophysiological study of superior colliculus that suggest input in the form of velocity being present in the saccadic system [18]. Our newly proposed velocity-based architecture adds credence to this neurophysiological observation. The finding of our study is also congruent with previous work which showed that the population activity in superior colliculus represents the intended movement trajectory [32]. The newly proposed model does not disprove any of the existing models which are based only on end-point displacement; alternately it suggests that the use of two different forms of desired inputs: (i) endpoint displacement, which is utilized as suggested by earlier models, and (ii) velocity, which is proposed by this new model; may both be viable algorithms to make the saccadic system more robust. In this regard, our study provides another example of how redundancy may be a general principle of the motor system. How and when the system chooses to use one form of information over the other is an interesting question that needs further investigation. Also, further experimental work would be required to establish the causal contribution of velocity-based control and distinguish this mechanism from endpoint control of saccades, which also explains the mean saccade trajectories.

Another feature of the saccadic system included in this study is the presence of noise, which has been neglected by many of the deterministic saccade models $[27,28]$. The use of velocity information at the internal feedback loop has been previously modeled to explain normal and blink perturbed saccade trajectories [33]. It was still in a deterministic setting without considering noise. The proposed stochastic model in this paper demonstrates the possible use of velocity control even when noise is present. However, the structure of the noise is assumed to be signal-dependent and acting additively on the optimal control signal that is given as input to the oculomotor plant. This noise structure was motivated by neurophysiological evidence provided in [23]. But there are further evidence suggesting that the saccadic system may minimize the consequences of motor noise, which was defined as a combination of the signal-dependent noise and constant noise [19]. Also, unlike a single source of noise assumed in this work at the motor command (control signal) level, there could be multiple sources of noise upstream in the saccadic system at the level of desired input and internal feedback level $[6,15]$. This opens further questions on the effect of variations in the structure of noise and possible sources of noise on the control architecture of saccades. Such studies may however require investigation of the variability in the saccade behavior rather than just the central tendencies.

An important aspect of optimal control models is the choice of the cost function that is minimized. In the proposed dynamic velocity model, we consider the cost to be comprised of the tracking error at each instant of time and the control effort. In contrast, the dynamic displacement model which was framed as an endpoint regulation problem had a cost function which was a combination of the error to the final state, the control effort, and the cost for each time-step. The error to the final state was weighted using a Heaviside function which came into effect only at the last time-step and the error weightage matrix $\left(Q_{k}\right)$ had to minimize errors in both the states, namely displacement and velocity to predict the trajectories of saccades. But in the newly proposed velocity-based model the error weightage matrix had to act only on the velocity errors. Correcting the velocity errors automatically predicted the saccade displacements accurately. Thus, compared to the dynamic displacement model which had two error weightage parameters that had to be estimated, the newly proposed model had only one error weightage 
parameter. However, since the estimated parameters were just one among the repertoire of multiple solutions that are possible, these parameters themselves do not appear to render any particular physiological interpretation. Additionally, the time-step cost was factored in by just simulating the models for the mean duration of saccades and this is a limitation of our model as the optimal duration of the saccades was not generated from the cost function. However, we speculate that the framework and results would still be valid with the time-step cost incorporated since the changes would be absorbed by a changed form of the recursive update equation of the weightage matrix $W_{k}$.

Apart from these theoretical implications to saccadic control, the model has much possible practical utilities in bioinspired engineering applications and clinical neurosciences. Algorithms developed based on saccadic control philosophy have been demonstrated to be useful in controlling devices like camera heads, autonomous indoor micro-flyers, and unmanned aerial vehicles for collision avoidance, hovering, steering, rapid video shooting technology, etc. [34-38]. The proposed model of saccade control may be useful for these applications. Saccadic eye movements are reported to have extensive clinical relevance in many neurological conditions that show deviations in saccade behavior from normal $[39,40]$. The proposed model may be leveraged in these conditions to design effective clinical interventions to help with early diagnosis as well as in improvising treatment protocols. For example, Parkinson's disease patients who are known to have lower levels of dopamine exhibit slowness of movement which in turn has been suggested to have a bearing on the optimal control policy of movements $[41,42]$. By implementing the effect of dopamine on the optimal control signal generated by our model, we could test whether the proposed velocity-based model can predict the effect of dopamine on eye movement velocities, to serve as a simple non-invasive biomarker of dopamine levels. However, these would require a detailed investigation of the model with the clinical data.

\section{Authors' contribution}

V. Varsha: Conceptualization, Methodology, Formal analysis, Investigation, Writing - Original Draft

Aditya Murthy: Conceptualization, Methodology, Resources, Writing - Review and editing

Radhakant Padhi: Conceptualization, Methodology, Resources, Writing - Review and editing

\section{Funding}

The experimental work was supported by grants from the Department of Biotechnology (DBT) - IISc partnership program Phase II (BT/ PR27952/INF/22/212/2018) and was carried out in Visuomotor lab, Centre for Neuroscience, Indian Institute of Science (IISc).

\section{Acknowledgements}

The authors are grateful to the participants of the experiment for their time and effort. The authors would like to thank Dr. Kapil Sachan, former PhD student, and Niranjan Chakrabhavi, PhD student, IISc for their valuable suggestions in enhancing the paper presentation.

\section{Declaration of Competing Interest}

The authors declare that they have no known competing financial interests or personal relationships that could have appeared to influence the work reported in this paper.

\section{References}

[1] B. Bridgeman, D. Hendry, L. Stark, Failure to detect displacement of the visual world during saccadic eye movements, Vis. Res. 15 (6) (1975) 719-722.

[2] D. Wolpert, Z. Ghahramani, Perception, planning and control of autonomous walking with the ambler planetary rover, Nat. Neurosci. 3 (2000) 1212-1217.

[3] E. Todorov, Optimality principles in sensorimotor control, Nat. Neurosci. 7 (9) (2004) 907-915.

[4] J.D. Enderle, J.W. Wolfe, Time-optimal control of saccadic eye movements, IEEE Trans. Biomed. Eng. (1) (1987) 43-55.

[5] A.A. Faisal, L.P. Selen, D.M. Wolpert, Noise in the nervous system, Nat. Rev. Neurosci. 9 (4) (2008) 292-303.

[6] R.J. van Beers, The sources of variability in saccadic eye movements, J. Neurosci. 7 (33) (2007) 8757-8770.

[7] E. Bollen, J. Bax, J. Van Dijk, M. Koning, J. Bos, C. Kramer, E. Van Der Velde, Variability of the main sequence, Investig. Ophthalmol. Visual Sci. 34 (13) (1993) 3700-3704.

[8] R. Jürgens, W. Becker, H. Kornhuber, Natural and drug-induced variations of velocity and duration of human saccadic eye movements: evidence for a control of the neural pulse generator by local feedback, Biol. Cybern. 39 (2) (1981) 87-96.

[9] G.L. West, T.N. Welsh, J. Pratt, Saccadic trajectories receive online correction: evidence for a feedback-based system of oculomotor control, J. Motor Behav. 41 (2) (2009) 117-127.

[10] T. Eggert, A. Straube, Saccade variability in healthy subjects and cerebellar patients, in: Progress in Brain Research, vol. 249, Elsevier, 2019, pp. 141-152.

[11] C.M. Harris, D.M. Wolpert, The main sequence of saccades optimizes speedaccuracy trade-off, Biol. Cybern. 95 (1) (2006) 21-29.

[12] E. Keller, N. Gandhi, J. Shieh, Endpoint accuracy in saccades interrupted by stimulation in the omnipause region in monkey, Visual Neurosci. 13 (1996) 1059-1068.

[13] B.A. Richardson, A. Ratneswaran, J. Lyons, R. Balasubramaniam, The time course of online trajectory corrections in memory-guided saccades, Exp. Brain Res. 212 (3) (2011) 457-469.

[14] M. Xu-Wilson, J. Tian, R. Shadmehr, D.S. Zee, Tms perturbs saccade trajectories and unmasks an internal feedback controller for saccades, J. Neurosci. 31 (32) (2011) 11537-11546.

[15] E. Todorov, Stochastic optimal control and estimation methods adapted to the noise characteristics of the sensorimotor system, Neural Comput. 17 (5) (2005) 1084-1108.

[16] R. Shadmehr, S. Mussa-Ivaldi, Biological Learning and Control: How the Brain Builds Representations, Predicts Events, and Makes Decisions, MIT Press, 2012.

[17] H. Chen-Harris, W.M. Joiner, V. Ethier, D.S. Zee, R. Shadmehr, Adaptive control of saccades via internal feedback, J. Neurosci. 28 (11) (2008) 2804-2813.

[18] I. Smalianchuk, U.K. Jagadisan, N.J. Gandhi, Instantaneous midbrain control of saccade velocity, J. Neurosci. 38 (47) (2018) 10156-10167.

[19] R.J. Van Beers, Saccadic eye movements minimize the consequences of motor noise, PloS ONE 3 (4) (2008) e2070.

[20] C.M. Harris, On the optimal control of behaviour: a stochastic perspective, J. Neurosci. Methods 83 (1) (1998) 73-88.

[21] D. Robinson, The use of control systems analysis in the neurophysiology of eye movements, Annu. Rev. Neurosci. 4 (1) (1981) 463-503.

[22] C.M. Harris, D.M. Wolpert, Signal-dependent noise determines motor planning, Nature 394 (6695) (1998) 780-784.

[23] K.E. Jones, A.F.d.C. Hamilton, D.M. Wolpert, Sources of signal-dependent noise during isometric force production, J. Neurophysiol. 8 (3) (2017) 1533-1544.

[24] D.S. Naidu, Optimal Control Systems, CRC Press, 2002.

[25] F.L. Lewis, D. Vrabie, V.L. Syrmos, Optimal Control, John Wiley \& Sons, 2012.

[26] A.T. Bahill, M.R. Clark, L. Stark, The main sequence, a tool for studying human eye movements, Math. Biosci. 24 (3) (1975) 191-204.

[27] D.A. Robinson, Models of the saccadic eye movement control system, Kybernetik 14 (2) (1973) 71-83.

[28] C.A. Scudder, A new local feedback model of the saccadic burst generator, J. Neurophysiol. 59 (5) (1988) 1455-1475.

[29] H. Aizawa, R.H. Wurtz, Reversible inactivation of monkey superior colliculus. i. curvature of saccadic trajectory, J. Neurophysiol. 79 (4) (1998) 2082-2096.

[30] D. Sparks, C. Lee, W. Rohrer, Population coding of the direction, amplitude, and velocity of saccadic eye movements by neurons in the superior colliculus, in: Cold Spring Harbor Symposia on Quantitative Biology, vol. 55, Cold Spring Harbor Laboratory Press, 1990, pp. 805-811.

[31] A. Berthoz, A. Grantyn, J. Droulez, Some collicular efferent neurons code saccadic eye velocity, Neurosci. Lett. 72 (3) (1986) 289-294.

[32] H. Goossens, A.J. van Opstal, Optimal control of saccades by spatial-temporal activity patterns in the monkey superior colliculus, PLoS Comput. Biol. 8 (5) (2012) e1002508.

[33] K. Arai, S. Das, E.L. Keller, E. Aiyoshi, A distributed model of the saccade system: simulations of temporally perturbed saccades using position and velocity feedback, Neural Netw. 12 (10) (1999) 1359-1375.

[34] J. Bruske, M. Hansen, L. Riehn, G. Sommer, Biologically inspired calibration-free adaptive saccade control of a binocular camera-head, Biol. Cybern. 77 (6) (1997) $433-446$. 
[35] J.-C. Zufferey, D. Floreano, Fly-inspired visual steering of an ultralight indoor aircraft, IEEE Trans. Robot. 22 (1) (2006) 137-146.

[36] A. Manecy, N. Marchand, S. Viollet, Hovering by gazing: a novel strategy for implementing saccadic flight-based navigation in gps-denied environments, Int. J. Adv. Robot. Syst. 11 (4) (2014) 66.

[37] J.P. Lindemann, H. Weiss, R. Möller, M. Egelhaaf, Saccadic flight strategy facilitates collision avoidance: closed-loop performance of a cyberfly, Biol. Cybern. 98 (3) (2008) 213-227.

[38] K. Iida, H. Oku, Saccade mirror 3: high-speed gaze controller with ultra wide gaze control range using triple rotational mirrors, in: 2016 IEEE International Conference on Robotics and Automation (ICRA), IEEE, 2016, pp. 624-629.
[39] R.J. Leigh, C. Kennard, Using saccades as a research tool in the clinical neurosciences, Brain 127 (3) (2004) 460-477.

[40] Y. Terao, H. Fukuda, O. Hikosaka, What do eye movements tell us about patients with neurological disorders?-an introduction to saccade recording in the clinical setting-, Proc. Jpn. Acad. Ser. B 93 (10) (2017) 772-801.

[41] D. Meder, D.M. Herz, J.B. Rowe, S. Lehéricy, H.R. Siebner, The role of dopamine in the brain-lessons learned from parkinson's disease, Neuroimage 190 (2019) 79-93.

[42] R. Shadmehr, J.W. Krakauer, A computational neuroanatomy for motor control, Exp. Brain Res. 185 (3) (2008) 359-381. 